\title{
Bound on vertical heat transport at large Prandtl number
}

\author{
Xiaoming Wang \\ Department of Mathematics, Florida State University, Tallahassee, FL 32306
}

October 27, 2007

\begin{abstract}
We prove a new upper bound on the vertical heat transport in Rayleigh-Bénard convection of the form $c R a^{\frac{1}{3}}(\ln R a)^{\frac{2}{3}}$ under the assumption that the ratio of Prandtl number over Rayleigh number satisfies $\frac{P r}{R a} \geq c_{0}$ where the non-dimensional constant $c_{0}$ depends on the aspect ratio of the domain only. This new rigorous bound agrees with the (optimal) $R a^{\frac{1}{3}}$ bound (modulo logarithmic correction) on vertical heat transport for the infinite Prandtl number model for convection due to Constantin and Doering [6] and Doering, Otto and Reznikoff [10]. It also improves a uniform (in Prandtl number) $R a^{\frac{1}{2}}$ bound for the Nusselt number [5] in the case of large Prandtl number.
\end{abstract}

keywords: Rayleigh-Bénard convection, Boussinesq equations, Prandtl number, Rayleigh number, Nusselt number

\section{Introduction}

We investigate the heat transport in the vertical direction in Rayleigh-Bénard convection at large Prandtl number. The governing system is the following classical Boussinesq system for Rayleigh-Bénard convection (non-dimensional):

$$
\begin{aligned}
\frac{1}{P r}\left(\frac{\partial \mathbf{u}}{\partial t}+(\mathbf{u} \cdot \nabla) \mathbf{u}\right)+\nabla p & =\Delta \mathbf{u}+\operatorname{Ra} \mathbf{k} T, \quad \nabla \cdot \mathbf{u}=0 \\
\frac{\partial T}{\partial t}+\mathbf{u} \cdot \nabla T & =\Delta T \\
\left.\mathbf{u}\right|_{z=0,1}= & 0 \\
\left.T\right|_{z=0}=1, & \left.T\right|_{z=1}=0 \\
\left.\mathbf{u}\right|_{t=0}=\mathbf{u}_{0}, & \left.T\right|_{t=0}=T_{0},
\end{aligned}
$$

where $\mathbf{u}=\left(u_{1}, u_{2}, u_{3}\right)$ is the fluid velocity field, $p$ is the kinetic pressure, $T$ is the temperature field, $\mathbf{k}$ is the unit upward vector, $R a$ is the Rayleigh number measuring the ratio of 
differential heating over overall dissipations, $\operatorname{Pr}$ is the Prandtl number which is the ratio of kinematic viscosity over thermal diffusivity, and the fluids occupy the (non-dimensionalized) region

$$
\Omega=\left[0, L_{x}\right] \times\left[0, L_{y}\right] \times[0,1]
$$

with periodicity in the horizontal directions assumed for simplicity.

At very large Prandtl number, we may formally set the Prandtl number to infinity and arrive at the following infinite Prandtl number model (non-dimensional)

$$
\begin{aligned}
\nabla p^{0} & =\Delta \mathbf{u}^{0}+R a \mathbf{k} T^{0}, \quad \nabla \cdot \mathbf{u}^{0}=0 \\
\frac{\partial T^{0}}{\partial t}+\mathbf{u}^{0} \cdot \nabla T^{0} & =\Delta T^{0} \\
\left.\mathbf{u}^{0}\right|_{z=0,1} & =0 \\
\left.T\right|_{z=0}=1, & \left.T\right|_{z=1}=0
\end{aligned}
$$

which is relevant for fluids such as silicone oil and the earth's mantle as well as many gases under high pressure $[3,12,2]$. One observes that the Navier-Stokes equations in the Boussinesq system has been replaced by the Stokes system in the infinite Prandtl number model.

The fact that the velocity field is linearly slaved by the temperature field has been exploited in several recent very interesting works on rigorous estimates on the rate of heat transport in the vertical direction in this infinite Prandtl number setting (see $[8,6,10]$ and the references therein, as well as the work of $[3,15])$. Recall that the Nusselt number is defined as the enhancement of heat transport in the vertical direction due to convection over conduction (see (32) below for a mathematical definition). In particular, a bound on the Nusselt number which scales like $R a^{\frac{1}{3}}$ (modulo logarithmic correction) was first rigorously obtained in [6] with the help of a maximum principle in the temperature field, and then also in [10] with an explicit prefactor and reduced logarithmic correction term. In the case of large Prandtl number, we can formally view the Boussinesq system as a small perturbation of the infinite Prandtl number model and hence the velocity field is almost linearly slaved by the temperature field. This near linear slavery was exploited in [29] in studying statistical properties of the Boussinesq system at large Prandtl number.

On the other hand, the best known bound on Nusselt number with arbitrary Prandtl number scales like $R a^{\frac{1}{2}}$ uniformly in $\operatorname{Pr} \in(0, \infty]$ [5] while the bound calculated via marginal stability [14] and some direct numerical simulations [1] suggest the same $R a^{\frac{1}{3}}$ scaling as the true behavior. We naturally ask if the $R a^{\frac{1}{3}}$ upper bound on the Nusselt number can be derived for at least the case of large but finite Prandtl number. As we shall demonstrate below, there exists a non-dimensional constant $c_{0}$ defined in (28) which depends on the aspect ratio of the domain only, such that $N u \leq c R a^{\frac{1}{3}}(R a)^{\frac{2}{3}}$ provided that the Prandtl number is relatively large compared to the Rayleigh number, i.e., $\frac{P r}{R a} \geq c_{0}$. This is a qualitative improvement over our previous result where a bound of the form of $R a^{\frac{1}{3}}(\ln R a)^{\frac{1}{3}}+\frac{R a^{\frac{7}{2}}}{P r^{2}}$ was 
derived [29]. The large Prandtl number condition (28) can be viewed as a small Reynolds number condition since the ratio of inertial over viscous term is proportional to $R a / P r$.

Throughout this manuscript, we assume the physically important case of high Rayleigh number $R a \gg 1$ so that we may have non-trivial dynamics.

We also follow the mathematical tradition of denoting our small parameter as $\varepsilon$, i.e.

$$
\varepsilon=\frac{1}{\operatorname{Pr}}
$$

Also $c$ will denote a generic non-dimensional constant independent of the Rayleigh number and Prandtl number throughout the manuscript.

The rest of the manuscript is organized as follows. In section 2 we recall a few a priori estimates on the solutions to the Boussinesq system at large Prandtl number. In section 3,

we derive the $R a^{\frac{1}{3}}$ upper bound for the Nusselt numbers at large Prandtl number. In section 4 , we offer concluding remarks.

\section{A priori estimates}

In this section we derive a few a priori estimates on solutions of the Boussinesq system at large Prandtl number. These estimates are essentially contained in [28] and [5] and we reproduce some of them here for the sake of completeness.

Throughout this manuscript, we will assume that the range of initial temperature $T_{0}$ is contained in the unit interval $[0,1]$. Hence we deduce by maximum principle that the range of $T$ in contained in $[0,1]$ for all time, i.e.,

$$
\|T\|_{L^{\infty}} \leq 1
$$

We will also assume that the background temperature profile $\tau(z)$ under consideration is always contained in the unit interval $[0,1]$ (see 41). Therefore, the perturbative temperature field $\theta=T-\tau$ satisfies the same estimate

$$
\|\theta\|_{L^{\infty}} \leq 1
$$

We also recall [5] that the following estimates hold

$$
\begin{aligned}
& <\|\nabla \mathbf{u}\|^{2}>\leq c R a^{\frac{3}{2}} \\
& <\|\nabla T\|^{2}>\leq c R a^{\frac{1}{2}}
\end{aligned}
$$

for all suitable weak solutions of the Boussinesq system with arbitrary Prandtl number where $<\cdot>$ represents long time average, i.e.

$$
<f(\cdot)>=\limsup _{t \rightarrow \infty} \frac{1}{t} \int_{0}^{t} f(s) d s .
$$


We first multiply the velocity equation (1) by $\mathbf{u}$ and integrate over the domain and we have

$$
\begin{aligned}
\frac{\varepsilon}{2} \frac{d}{d t}\|\mathbf{u}(t)\|_{L^{2}}^{2}+\|\nabla \mathbf{u}(t)\|^{2} & \leq R a\|T(t)\|\left\|u_{3}(t)\right\| \\
& \leq \frac{1}{2 \pi^{2}} R a^{2}|\Omega|+\frac{\pi^{2}}{2}\|\mathbf{u}(t)\|^{2}
\end{aligned}
$$

and hence

$$
\frac{\varepsilon}{2} \frac{d}{d t}\|\mathbf{u}(t)\|_{L^{2}}^{2}+\frac{\pi^{2}}{2}\|\mathbf{u}(t)\|^{2} \leq \frac{1}{2 \pi^{2}} R a^{2}|\Omega|
$$

where we have utilized the maximum principle on $T$, Poincaré inequality and Cauchy-Schwarz inequality. We then apply Gronwall inequality to deduce

$$
\limsup _{t \rightarrow \infty}\|\mathbf{u}(t)\| \leq R a|\Omega|^{\frac{1}{2}} / \pi^{2} .
$$

Next we multiply the velocity equation (1) by $A \mathbf{u}$ (where $A$ denotes the Stokes operator with viscosity one and the associated boundary conditions), integrate over the domain and we have

$$
\begin{aligned}
\frac{\varepsilon}{2} \frac{d}{d t}\|\nabla \mathbf{u}\|^{2}+\|A \mathbf{u}\|^{2} & \leq R a\|T\|\|A \mathbf{u}\|+\varepsilon\|\nabla \mathbf{u}\|\|A \mathbf{u}\|\|\mathbf{u}\|_{L^{\infty}} \\
& \leq R a|\Omega|^{\frac{1}{2}}\|A \mathbf{u}\|+c_{A} \varepsilon\|\nabla \mathbf{u}\|^{\frac{3}{2}}\|A \mathbf{u}\|^{\frac{3}{2}} \\
& \leq \frac{1}{2}\|A \mathbf{u}\|^{2}+R a^{2}|\Omega|+64 c_{A}^{4} \varepsilon^{4}\|\nabla \mathbf{u}\|^{6}
\end{aligned}
$$

where $c_{A}$ is the constant in the Agmon inequality $\|\mathbf{u}\|_{L^{\infty}} \leq c_{A}\|\nabla \mathbf{u}\|^{\frac{1}{2}}\|A \mathbf{u}\|^{\frac{1}{2}}$. This implies, together with Poincaré inequality,

$$
\varepsilon \frac{d}{d t}\|\nabla \mathbf{u}\|^{2}+\pi^{2}\|\nabla \mathbf{u}\|^{2} \leq 2|\Omega| R a^{2}+128 c_{A}^{4} \varepsilon^{4}\|\nabla \mathbf{u}\|^{6}
$$

It is then easy to verify that the ball of radius $2|\Omega|^{\frac{1}{2}} R a / \pi$ is invariant under the dynamics if the following relative large Prandtl number condition holds

$$
\frac{\operatorname{Pr}}{R a} \geq 8 c_{A}|\Omega|^{\frac{1}{2}} / \pi^{\frac{3}{2}}
$$

On the other hand, estimate (14) implies that any orbit should enter this ball of radius $2|\Omega|^{\frac{1}{2}} R a / \pi$. Hence this is an absorbing ball and we have the estimate

$$
\limsup _{t \rightarrow \infty}\|\nabla \mathbf{u}(t)\| \leq 2 R a|\Omega|^{\frac{1}{2}} / \pi
$$

Inserting this into (18) and taking the long time average, we have the following estimate

$$
<\|A \mathbf{u}\|^{2}>\leq c R a^{2}|\Omega| .
$$


Next, we estimate the time derivative of the velocity. For this purpose we differentiate the velocity equation (1) in time ${ }^{1}$ and deduce

$$
\varepsilon\left(\frac{\partial^{2} \mathbf{u}}{\partial t^{2}}+\left(\frac{\partial \mathbf{u}}{\partial t} \cdot \nabla\right) \mathbf{u}+(\mathbf{u} \cdot \nabla) \frac{\partial \mathbf{u}}{\partial t}\right)+\nabla \frac{\partial p}{\partial t}=\Delta \frac{\partial \mathbf{u}}{\partial t}+R a \mathbf{k} \frac{\partial T}{\partial t} .
$$

Multiplying this equation by $\frac{\partial \mathbf{u}}{\partial t}$ and integrating over $\Omega$, we deduce, for large $t$,

$$
\begin{aligned}
\frac{\varepsilon}{2} \frac{d}{d t}\left\|\frac{\partial \mathbf{u}}{\partial t}\right\|^{2}+\left\|\nabla \frac{\partial \mathbf{u}}{\partial t}\right\|^{2} & \leq R a\left\|\frac{\partial T}{\partial t}\right\|_{H^{-1}}\left\|\nabla \frac{\partial \mathbf{u}}{\partial t}\right\|+\varepsilon\|\mathbf{u}\|_{L^{3}}\left\|\nabla \frac{\partial \mathbf{u}}{\partial t}\right\|\left\|\frac{\partial \mathbf{u}}{\partial t}\right\|_{L^{6}} \\
& \leq R a\left\|\frac{\partial T}{\partial t}\right\|_{H^{-1}}\left\|\nabla \frac{\partial \mathbf{u}}{\partial t}\right\|+c_{S} \varepsilon\|\nabla \mathbf{u}\|^{\frac{1}{2}}\|\mathbf{u}\|^{\frac{1}{2}}\left\|\nabla \frac{\partial \mathbf{u}}{\partial t}\right\|^{2} \\
& \leq \frac{1}{4}\left\|\nabla \frac{\partial \mathbf{u}}{\partial t}\right\|^{2}+R a^{2}\left\|\frac{\partial T}{\partial t}\right\|_{H^{-1}}^{2}+\frac{2}{\pi^{\frac{3}{2}}} R a|\Omega|^{\frac{1}{2}} c_{S} \varepsilon\left\|\nabla \frac{\partial \mathbf{u}}{\partial t}\right\|^{2}
\end{aligned}
$$

where $c_{S}=c_{S 1} c_{S 2}$ is the product of two Sobolev constants in $\|\mathbf{u}\|_{L^{3}} \leq c_{S 1}\|\nabla \mathbf{u}\|^{\frac{1}{2}}\|\mathbf{u}\|^{\frac{1}{2}}$ and $\left\|\frac{\partial \mathbf{u}}{\partial t}\right\|_{L^{6}} \leq c_{S 2}\left\|\nabla \frac{\partial \mathbf{u}}{\partial t}\right\|^{2}$.

This implies that we have

$$
<\left\|\nabla \frac{\partial \mathbf{u}}{\partial t}\right\|^{2}>\leq 2 R a^{2}<\left\|\frac{\partial T}{\partial t}\right\|_{H^{-1}}^{2}>
$$

provided the following second relative large Prandtl number condition is satisfied

$$
\frac{\operatorname{Pr}}{R a} \geq 8 c_{S}|\Omega|^{\frac{1}{2}} / \pi^{\frac{3}{2}}
$$

Setting

$$
c_{0}=\frac{8}{\pi^{\frac{3}{2}}} \max \left\{c_{A}, c_{S}\right\}|\Omega|^{\frac{1}{2}}
$$

we can combine the two relative large Prandtl number conditions (20) and (26) into the following one relative large Prandtl number condition

$$
\frac{\operatorname{Pr}}{R a} \geq c_{0}
$$

Next, we utilize the temperature equation (2) to deduce

$$
\begin{aligned}
\left\|\frac{\partial T}{\partial t}\right\|_{H^{-1}} & \leq\|T \mathbf{u}\|+\|\nabla T\| \\
& \leq \frac{1}{\pi}\|\nabla \mathbf{u}\|+\|\nabla T\|
\end{aligned}
$$

where we have used the maximum principle on the temperature field $T$ and the Poincaré inequality. This further implies, thanks to (14) and (15),

$$
<\left\|\frac{\partial T}{\partial t}\right\|_{H^{-1}}^{2}>\leq 2<\frac{1}{\pi^{2}}\|\nabla \mathbf{u}\|^{2}+\|\nabla T\|^{2}>\leq c R a^{\frac{3}{2}} .
$$

\footnotetext{
${ }^{1}$ We do not need $H^{1}$ norm of the time derivative. However, we do not know of good estimates on the $L^{2}$ or $H^{-1}$ norm of the time derivative.
} 
Therefore, inserting this into (25) we have

$$
<\left\|\nabla \frac{\partial \mathbf{u}}{\partial t}\right\|^{2}>\leq c R a^{\frac{7}{2}}
$$

\section{Bound on Nusselt number}

We first recall the definition of the long time averaged Nusselt number measuring the heat transport in the vertical direction

$$
\begin{aligned}
(N u)_{\varepsilon} & =\sup _{\left(\mathbf{u}_{0}, \theta_{0}\right) \in X} \limsup _{t \rightarrow \infty} \frac{1}{t L_{x} L_{y}} \int_{0}^{t} \int_{\Omega}|\nabla T(\mathbf{x}, s)|^{2} d \mathbf{x} d s \\
& =1+\sup _{\left(\mathbf{u}_{0}, \theta_{0}\right) \in X} \limsup _{t \rightarrow \infty} \frac{1}{t L_{x} L_{y}} \int_{0}^{t} \int_{\Omega} u_{3}(\mathbf{x}, s) T(\mathbf{x}, s) d \mathbf{x} d s \\
& =1+\sup _{\left(\mathbf{u}_{0}, \theta_{0}\right) \in X} \limsup _{t \rightarrow \infty} \frac{1}{t L_{x} L_{y}} \int_{0}^{t} \int_{\Omega} u_{3}(\mathbf{x}, s) \theta(\mathbf{x}, s) d \mathbf{x} d s
\end{aligned}
$$

where $T=1-z+\theta$ is the temperature field, and $(\mathbf{u}, \theta)$ are suitable weak solutions to the Boussinesq system with initial data $\left(\mathbf{u}_{0}, \theta_{0}\right)$. The Nusselt number is a statistical property of the Boussinesq system in the sense that it is the average of $1+u_{3} \theta$ over the whole phase space with respect to some appropriate invariant measure (stationary statistical solution) of the Boussinesq system [29].

The approach we take here is to view the Boussinesq system at large Prandtl number as a perturbation of the infinite Prandtl number model for convection. More specifically, we write the Boussinesq system as

$$
\begin{aligned}
\nabla p & =\Delta \mathbf{u}+R a \mathbf{k} \theta+\varepsilon \mathbf{f}, \quad \nabla \cdot \mathbf{u}=0 \\
\frac{\partial \theta}{\partial t}+\mathbf{u} \cdot \nabla \theta+u_{3} \tau^{\prime}(z) & =\Delta \theta+\tau^{\prime \prime}(z) \\
\left.\mathbf{u}\right|_{z=0,1} & =0 \\
\left.\theta\right|_{z=0,1} & =0 \\
\mathbf{f} & =-\left(\frac{\partial \mathbf{u}}{\partial t}+(\mathbf{u} \cdot \nabla) \mathbf{u}\right)
\end{aligned}
$$

where we have employed the background temperature profile method for the Boussinesq system proposed by Constantin and Doering $[5,6,10]$ which is a generalization of E. Hopf's original idea [25]. Here we diverge from our previous work [29] where we emphasized the positive contribution of the transport term $\int_{\Omega} u_{3} \theta$ (following the idea of [10]) with $u_{3}$ containing the effect of total derivative. As we shall see that our new approach of isolating the effect of total derivative provides better (optimal) estimate on the Nusselt number than lumping the total derivative term in the "asymptotic" spectral constraint approach that we used before.

Multiplying the temperature equation by $\theta$ and integrate over $\Omega$ we have

$$
\frac{1}{2} \frac{d}{d t}\|\theta\|^{2}+\|\nabla \theta\|^{2}+\left(\tau^{\prime}, \theta\right)+\int_{\Omega} \tau^{\prime} u_{3} \theta=0
$$


We also have

$$
\|\nabla T\|^{2}=\|\nabla \theta\|^{2}+2\left(\tau^{\prime}, \theta\right)+\left\|\tau^{\prime}\right\|^{2} .
$$

Following $[6,10]$ and combining the two we have

$$
\begin{aligned}
& <\|\nabla T\|^{2}> \\
= & \left\|\tau^{\prime}\right\|^{2}-<\int_{\Omega}\left(|\nabla \theta|^{2}+2 \tau^{\prime} u_{3} \theta\right)> \\
= & \left\|\tau^{\prime}\right\|^{2}-<\int_{\Omega}\left(|\nabla \theta|^{2}+2 \operatorname{Ra}^{\prime} A^{-1}(\mathbf{k} \theta)_{3} \theta+2 \varepsilon \tau^{\prime} A^{-1}(\mathbf{f})_{3} \theta\right)> \\
= & \left\|\tau^{\prime}\right\|^{2}-<\int_{\Omega}\left(|\nabla \theta|^{2}+2 \operatorname{Ra\tau ^{\prime }} A^{-1}(\mathbf{k} \theta)_{3} \theta-2 \varepsilon \tau^{\prime} A^{-1}\left(\frac{\partial \mathbf{u}}{\partial t}\right)_{3} \theta-2 \varepsilon \tau^{\prime} A^{-1}((\mathbf{u} \cdot \nabla) \mathbf{u})_{3} \theta\right)
\end{aligned}
$$

We now choose a specific background temperature profile of the form

$$
\tau^{\prime}(z)= \begin{cases}\frac{1}{\delta}, & 1-\delta \leq z \leq 1 \\ 0, & 0 \leq z \leq 1-\delta\end{cases}
$$

According to [6], we have

$$
\int_{\Omega}\left(\frac{1}{2}|\nabla \theta|^{2}+2 R a \tau^{\prime} A^{-1}(\mathbf{k} \theta)_{3} \theta\right) \geq 0, \quad \forall \theta
$$

provided we choose $\delta$ as

$$
\delta^{-1}=c_{C D} R a^{\frac{1}{3}}(1+\ln R a)^{\frac{2}{3}}
$$

for some appropriate constant $c_{C D}$ independent of the Rayleigh number. Roughly speaking, the idea of Constantin and Doering was to apply Poincaré inequality and to dominate $\left\|\frac{\partial^{2}}{\partial z^{2}} A^{-1}(\mathbf{k} \theta)_{3}\right\|_{L^{\infty}}=\left\|\frac{\partial^{2}}{\partial z^{2}} A^{-1}(\mathbf{k} T)_{3}\right\|_{L^{\infty}}$ by $\|T\|_{L^{\infty}}$ (which can be dominated by the maximum principle (12)) together with a logarithmic term in $\|\Delta T\|$ which induces the logarithmic term in $R a$. A polynomial in $R a$ bound on $\|\Delta T\|$ may be found in [28].

Now we have

$$
\begin{aligned}
2 \varepsilon\left|\int_{\Omega} \tau^{\prime} A^{-1}\left(\frac{\partial \mathbf{u}}{\partial t}\right)_{3} \theta\right| & \leq 2 \delta^{2} \varepsilon\left\|\frac{\partial^{2}}{\partial z^{2}} A^{-1}\left(\frac{\partial \mathbf{u}}{\partial t}\right)_{3}\right\|\left\|\frac{\partial \theta}{\partial z}\right\| \\
& \leq \frac{1}{4}\|\nabla \theta\|^{2}+c \delta^{4} \varepsilon^{2}\left\|\frac{\partial \mathbf{u}}{\partial t}\right\|^{2}
\end{aligned}
$$

where we have applied Poincaré, Hölder, Cauchy-Schwarz inequality, Stokes regularity, the boundary conditions for $\theta, A^{-1}\left(\frac{\partial \mathbf{u}}{\partial t}\right)_{3}$ and $\nabla A^{-1}\left(\frac{\partial \mathbf{u}}{\partial t}\right)_{3}$, and the specific form of $\tau(41)$.

We also have

$$
\begin{aligned}
2 \varepsilon\left|\int_{\Omega} \tau^{\prime} A^{-1}((\mathbf{u} \cdot \nabla) \mathbf{u})_{3} \theta\right| & \leq 2 \delta \varepsilon\left\|\frac{\partial^{2}}{\partial z^{2}} A^{-1}((\mathbf{u} \cdot \nabla) \mathbf{u})_{3}\right\|\|\theta\| \\
& \leq c \delta \varepsilon\|(\mathbf{u} \cdot \nabla) \mathbf{u}\|
\end{aligned}
$$

where we have applied Poincaré inequality, Stokes regularity, the specific form for $\tau(41)$ and the maximum principle on $\theta$. 
Therefore we deduce

$$
\begin{aligned}
& <\|\nabla T\|^{2}> \\
\leq & \left\|\tau^{\prime}\right\|^{2}-<\int_{\Omega}\left(\frac{1}{2}|\nabla \theta|^{2}+2 R a \tau^{\prime} A^{-1}(\mathbf{k} \theta)_{3} \theta\right)>+c \delta^{4} \varepsilon^{2}<\left\|\frac{\partial \mathbf{u}}{\partial t}\right\|^{2}>+c \delta \varepsilon<\|(\mathbf{u} \cdot \nabla) \mathbf{u}\|> \\
\leq & \left\|\tau^{\prime}\right\|^{2}+c \delta^{4} \varepsilon^{2}<\left\|\frac{\partial \mathbf{u}}{\partial t}\right\|^{2}>+c \delta \varepsilon<\|(\mathbf{u} \cdot \nabla) \mathbf{u}\|> \\
\leq & \left\|\tau^{\prime}\right\|^{2}+c \delta^{4} \varepsilon^{2}<\left\|\nabla \frac{\partial \mathbf{u}}{\partial t}\right\|^{2}>+c \delta \varepsilon<\|\mathbf{u}\|_{L^{\infty}}\|\nabla \mathbf{u}\|> \\
\leq & \left\|\tau^{\prime}\right\|^{2}+c \delta^{4} \varepsilon^{2}<\left\|\nabla \frac{\partial \mathbf{u}}{\partial t}\right\|^{2}>+c \delta \varepsilon<\|\nabla \mathbf{u}\|^{\frac{3}{2}}\|A \mathbf{u}\|^{\frac{1}{2}}> \\
\leq & \left\|\tau^{\prime}\right\|^{2}+c \delta^{4} \varepsilon^{2}<\left\|\nabla \frac{\partial \mathbf{u}}{\partial t}\right\|^{2}>+c \delta \varepsilon<\|\nabla \mathbf{u}\|^{2}>>^{\frac{3}{4}}<A \mathbf{u} \|^{2}>^{\frac{1}{4}} \\
\leq & c R a^{\frac{1}{3}}(\ln R a)^{\frac{2}{3}}+c \varepsilon^{2} R a^{\frac{13}{6}}(\ln R a)^{-\frac{8}{3}}+c \varepsilon R a^{\frac{31}{24}}(\ln R a)^{-\frac{2}{3}} \\
\leq & c R a^{\frac{1}{3}}(\ln R a)^{\frac{2}{3}}+c R a^{\frac{1}{6}}(\ln R a)^{-\frac{8}{3}}+c R a^{\frac{7}{24}}(\ln R a)^{-\frac{2}{3}} \\
\leq & c R a^{\frac{1}{3}}(\ln R a)^{\frac{2}{3}}
\end{aligned}
$$

where we have utilized the spectral constraint (42), Poincaré inequality, Agmon inequality, Hölder's inequality, the specific form of $\tau$ and our choice of $\delta$ together with the a priori estimates $(14,22,31)$ as well as the large Prandtl number assumption (28). This completes the proof of our main result.

\section{Concluding Remarks}

We have derived a new upper bound on the Nusselt number (non-dimensional measurement of heat transport in the vertical direction) for the Boussinesq system under the relatively large Prandtl number over Rayleigh number assumption (28). The new bound (46) scales

like $R a^{\frac{1}{3}}$ (modulo logarithmic term) in agreement with the best known upper bounds on the Nusselt number for the infinite Prandtl number model derived earlier by Constantin and Doering [6], and Doering, Otto and Reznikoff [10] again. The result here is optimal (modulo logarithmic correction) if one believes that the $R a^{\frac{1}{3}}$ scaling is optimal for the infinite Prandtl number model of Rayleigh-Bénard convection.

The large Prandtl number assumption (28) may be interpreted as a small Reynolds number assumption since the ratio of the inertial and viscous terms which we interpreted as the Reynolds number is heuristically proportional to $\frac{R a}{P r}$ (for fixed Rayleigh number) at large Prandtl number. An alternative interpretation due to Charlie Doering is to notice that $\frac{R a}{P r}=R e_{\max }^{2}$ where the maximum Reynolds number is defined through the maximum free-fall velocity $U_{\text {max }} \sim(g \alpha \Delta T h)^{\frac{1}{2}}$ where $g$ is the acceleration of gravity, $\alpha$ is the thermal expansion coefficient of the fluid, $\Delta T$ is the temperature gap between the bottom and top plates, and $h$ is the distance between the two plates. Hence relative large Prandtl number over Rayleigh number is exactly small maximum Reynolds number assumption. This small 


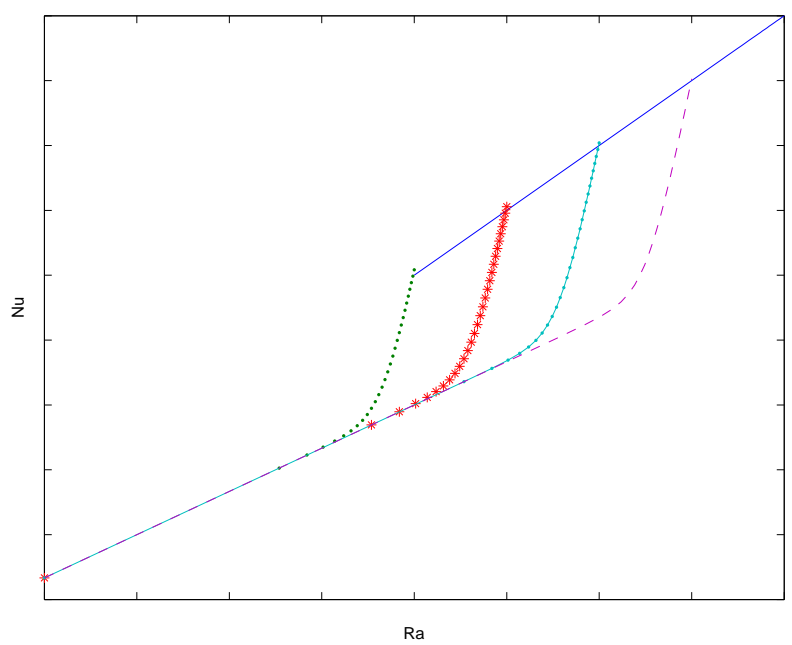

Figure 1: Schematic log-log plot of the new upper bound on the Nusselt number (46) in junction with the uniform $R a^{\frac{1}{2}}$ bound versus Rayleigh number for different values of the Prandtl number.

Reynolds number assumption is also in accordance with the belief that the $R a^{\frac{1}{2}}$ scaling can only occur at large Reynolds number region when the flow is turbulent.

The result is derived via viewing the Boussinesq system at large Prandtl number as a small (singular) perturbation of the infinite Prandtl number model. The perturbation idea works for this singular perturbation case [27] since this special two time scale problem is of relaxation type and we are looking for long time (stationary) behavior [29]. The perturbative approach also allows us to improve bound on the Nusselt number for the Boussinesq system if better bounds on the Nusselt number for the infinite Prandtl number are derived.

We point out that whether or not the $R a^{\frac{1}{3}}$ scaling (modulo logarithmic corrections) of the Nusselt number is true for all Prandtl number (when the large Prandtl number assumption is violated) is still an outstanding open problem.

\section{Acknowledgments}

Part of the work was completed when the author was a visitor at the Institute for Applied Mathematics at Bonn University. The financial support and hospitality of the Institute is gratefully acknowledged. The author also acknowledges stimulating conversation with Charlie Doering. The work is supported in part by grants from the National Science Foundation.

\section{References}

[1] Amati, G., Koal, K., Massaioli, F., Sreenivasan, K.R., and Verzicco, R., Turbulent thermal convection at large Rayleigh numbers for a Boussinesq fluid of constant Prandtl 
number, Physics of Fluids, 17 (12), 2005.

[2] Bodenschatz, E.; Pesch, W.; Ahlers, G.; Recent developments in Rayleigh-Bnard convection. Annual review of fluid mechanics, Vol. 32, 709-778, 2000.

[3] Busse, F.H.; Fundamentals of thermal convection. In Mantle Convection: Plate Tectonics and Global Dynamics, ed WR Peltier, pp. 23-95. Montreux: Gordon and Breach, 1989.

[4] Chandrasekhar, S.; Hydrodynamic and hydro-magnetic stability. Oxford, Clarendon Press, 1961.

[5] Constantin, P.; Doering, C.R.; Heat transfer in convective turbulence, Nonlinearity, 9, (1996), pp.1049-1060.

[6] Constantin, P.; Doering, C.R.; Infinite Prandtl number convection, J. Stat. Phys. 94 (1999), no. 1-2, 159-172.

[7] Constantin, P.; Foias, C.; Navier-Stokes Equations, Chicago University Press.

[8] Doering, C.R.; Constantin, P.; On upper bounds for infinite Prandtl number convection with or without rotation, J. Math. Phys. 42, no. 2, 784-795, 2001.

[9] Doering, C.R.; Gibbon, J.D.; Applied Analysis of the Navier-Stokes Equations, Cambridge University Press, Cambridge, UK.

[10] Doering, C.R.; Otto, F.; Reznikoff, M.G., Bounds on vertical heat transport for infinite Prandtl number Rayleigh-Bénard convection, J. Fluid Mech. (2006), vol. 560, pp.229241.

[11] Foias, C.; Manley, O.; Rosa, R.; Temam, R.; Navier-Stokes equations and turbulence. Encyclopedia of Mathematics and its Applications, 83. Cambridge University Press, Cambridge, 2001.

[12] Getling, A. V.; Rayleigh-Bénard convection. Structures and dynamics. Advanced Series in Nonlinear Dynamics, 11. World Scientific Publishing Co., Inc., River Edge, NJ, 1998.

[13] Grossmann, S.; Lohse, D.; Scaling in thermal convection: a unifying theory, J. Fluid Mech., vol. 407, 2000, pp.27-56.

[14] Howard, L.; Heat transport by turbulent convection, J. Fluid Mech. 17, 1963, pp.405-432.

[15] Ierley, G.R.; Kerswell, R.R. and Plasting, S.C., Infinite-Prandtl-number convection. Part 2. A singular limit of upper bound theory, J. Fluid Mech. (2006), vol. 560, pp.157-227.

[16] Kadanoff, L.P., Turbulent heat flow: structures and scaling, Physics Today, 54, no. 8, pp.34-39, 2001. 
[17] Lax, P.D., Functional Analysis, New York: Wiley, c2002.

[18] Majda, A.J., and Bertozzi, A., Vorticity and Incompressible Flow, Cambridge University Press, Cambridge, England, 2001.

[19] Majda, A.J. and Wang, X., Nonlinear Dynamics and Statistical Theory for Basic Geophysical Flows, Cambridge University Press, Cambridge, England, (2006).

[20] Monin, A.S.; Yaglom, A.M., Statistical fluid mechanics; mechanics of turbulence, English ed. updated, augmented and rev. by the authors. MIT Press, Cambridge, Mass., 1975.

[21] Nikolaenko, A., Brown, E., Funfschilling D., and Ahlers G., Heat transport by turbulent Rayleigh-Benard convection in cylindrical cells with aspect ratio one and less. J. Fluid Mech. (2005), vol. 523, pp. 251-260.

[22] Siggia, E.; High Rayleigh number convection. Annual review of fluid mechanics, Vol. 26, 137-168, Annual Reviews, Palo Alto, CA, 1994.

[23] Temam, R.M.; Navier-Stokes equations and nonlinear functional analysis, 2nd ed. Philadelphia, Pa. : SIAM, 1995.

[24] Temam, R.M.; Infinite Dimensional Dynamical Systems in Mechanics and Physics, 2nd ed, Springer-Verlag, New York, 1997.

[25] Temam, R.M.; Navier-Stokes Equations, AMS Chelsea, Providence, Rhode Island, 2000.

[26] Vishik, M.I.; Fursikov, A.V., Mathematical Problems of Statistical Hydromechanics, Kluwer Acad. Publishers, Dordrecht/Boston/London, 1988.

[27] Wang, X.; Infinite Prandtl Number Limit of Rayleigh-Bénard Convection, CommuniCations on Pure and Applied Mathematics, vol 57, issue 10, (2004), 1265-1282.

[28] Wang, X.; Asymptotic behavior of global attractors to the Boussinesq system for Rayleigh-Bénard convection at large Prandtl number, Communications on PURE AND Applied Mathematics, vol 60, issue 9, (2007), 1293-1318.

[29] Wang, X.; Stationary statistical properties of Rayleigh-Bénard convection at large Prandtl number, CPAM, accepted for publication. 